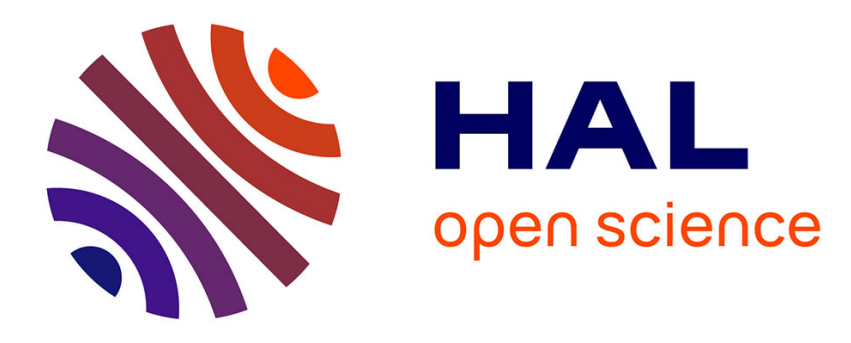

\title{
On the multiple-humped fission barriers and half-lives of actinides
}

C. Bonilla, Guy Royer

\section{To cite this version:}

C. Bonilla, Guy Royer. On the multiple-humped fission barriers and half-lives of actinides. Heavy Ion Physics, 2006, 25, pp.11-28. in2p3-00025719

\section{HAL Id: in2p3-00025719 \\ https://hal.in2p3.fr/in2p3-00025719}

Submitted on 2 Mar 2006

HAL is a multi-disciplinary open access archive for the deposit and dissemination of scientific research documents, whether they are published or not. The documents may come from teaching and research institutions in France or abroad, or from public or private research centers.
L'archive ouverte pluridisciplinaire HAL, est destinée au dépôt et à la diffusion de documents scientifiques de niveau recherche, publiés ou non, émanant des établissements d'enseignement et de recherche français ou étrangers, des laboratoires publics ou privés. 
Acta Phys. Hung. A 25/1 (2006) 000-000

HEAVY ION

PHYSICS

\title{
On the Multiple-Humped Fission Barriers and Half-Lives of Actinides
}

\author{
C. Bonilla and G. Royer \\ Laboratoire Subatech, UMR: IN2P3/CNRS - Université - Ecole des Mines \\ 4 rue A. Kastler, 44307 Nantes Cedex 03, France
}

Received 4 March 2005

\begin{abstract}
The energy of actinide nuclei has been determined within a generalized liquid drop model taking into account the proximity energy, the mass and charge asymmetry, an accurate nuclear radius in adding the shell and pairing energies. Double and triple-humped potential barriers appear. The second maximum corresponds to the transition from compact and creviced one-body shapes to two touching ellipsoids. A third minimum and third peak appear in special asymmetric exit channels where one fragment is almost a magic nucleus with a quasi-spherical shape while the other one evolves from oblate to prolate shapes. The heights of the double and triple-humped fission barriers agree precisely with the experimental results in all the actinide region. The predicted half-lives follow the experimental data trend.
\end{abstract}

Keywords: fission, actinides, Liquid Drop Model, half-lives

PACS: 24.75.+i, 21.60.Ev, 27.90.+b

\section{Introduction}

The possibility of transmutation of nuclear waste and of production of energy by accelerator-driven systems is under consideration. The knowledge of all the nuclear reactions which constitute a non-negligible part of the reaction cross section is needed [1]. Different codes are under construction or improvement (Fluka, Gnash, Talys, ...). Accurate potential barriers must be calculated rapidly, particularly in the actinide region, to predict the fission cross sections. Furthermore, new measurements renew also interest in investigating the multiple-humped barriers of the actinide nuclei and heaviest elements. The analysis of the fission probability and of the angular distribution of the fission fragments support the presence of hyperdeformed states in a deep third well in several Th and U isotopes [2-4] confirming the pioneering work of Blons et al. [5] in ${ }^{231,233} \mathrm{Th}$. The observed strongly enhanced low 
energy $\alpha$ decay in some heavy actinide nuclei is also explained by transition from a third hyperdeformed minimum and the possibility that the third minimum is the true ground state of very heavy and perhaps superheavy nuclei is even also advocated [6]. In medium mass nuclei some signs of hyperdeformed rotational bands have been found, but no discrete HD level has been identified up to now $[7,8]$.

By adding at the macroscopic liquid drop model energy of elongated one-body shapes an oscillatory microscopic contribution, the Strutinsky's method [9] generated double-humped barriers allowing to explain and predict partially the fission isomer characteristics. Analytic expressions have also been proposed [10,11] in the same objective of taking into account the shell and pairing energies. Later on, the asymmetric two-center shell model [13], Hartree-Fock-Bogoliubov [14] and relativistic mean field theories [15] have also partially succeeded, for some specific isotopes, in obtaining several energy minima where superdeformed fission isomers can survive. The whole reproduction of the heights of the inner and outer asymmetric fission barriers which are almost constant (5-6 MeV) from Th to Am isotopes $[16,17]$ is a very difficult challenge.

It has been previously shown within a Generalized Liquid Drop Model taking into account both the proximity energy between close opposite surfaces, the mass and charge asymmetry and an accurate radius that the proximity forces strongly lower the deformation energy of compact and creviced shapes and allow also to obtain the experimental fission barrier heights in the whole mass range [18]. Recently, the asymmetric fission barrier heights for the $\mathrm{Se}, \mathrm{Br}, \mathrm{Mo}$, In, Tb and $\mathrm{Hg}$ nuclei have also been reproduced with the GLDM $[19,20]$. Within the same approach, the $\alpha$ and cluster emission [21-23] as well as the super and highly deformed state [24] and fusion [25] data can also be reproduced.

The purpose of this work is to focus on the actinide region taking into account the ellipsoidal deformations of the two different fission fragments and their associated shell and pairing energies, in investigating all the possible mass and charge asymmetries.

\section{Potential Energy}

The total energy of a deformed nucleus is the sum of the GLDM energy and the shell and pairing energies. The GLDM energy is given by [25]

$$
E=E_{V}+E_{S}+E_{C}+E_{\text {prox }}+E_{\text {rot }},
$$

where the different terms are respectively the volume, surface, Coulomb, nuclear proximity and rotational energies.

All along the deformation path the nuclear proximity energy term $E_{\text {prox }}$ allows to take into account the effects of the attractive nuclear forces between nucleons facing each other across a neck in the case of a deformed one-body shape or across a gap in the case of two separated fragments. This is not a small correction for compact and creviced shapes. For example, at the contact point between two spherical 
$\mathrm{Kr}$ and $\mathrm{Ba}$ nuclei the proximity energy reaches $-43 \mathrm{MeV}$

$$
E_{\text {prox }}(r)=2 \gamma \int \Phi[D(r, h) / b] 2 \pi h d h .
$$

$r$ is the distance between the mass centres. $h$ is the transverse distance varying from the neck radius to the height of the neck border. $D$ is the distance between the opposite surfaces and $b$ the surface width. $\Phi$ is the proximity function and $\gamma$ the surface parameter.

\subsection{One-body shapes}

For one-body shapes, the first three contributions are given by

$$
\begin{aligned}
& E_{V}=-15.494\left(1-1.8 I^{2}\right) A \mathrm{MeV}, \\
& E_{S}=17.9439\left(1-2.6 I^{2}\right) A^{2 / 3} \frac{S}{4 \pi R_{0}^{2}} \mathrm{MeV}, \\
& E_{C}=0.6 e^{2}\left(Z^{2} / R_{0}\right) B_{C} .
\end{aligned}
$$

$B_{C}$ is the Coulomb shape dependent function, $S$ is the surface and $I$ is the relative neutron excess [25].

$$
B_{C}=0.5 \int\left(V(\theta) / V_{0}\right)\left(R(\theta) / R_{0}\right)^{3} \sin \theta d \theta
$$

where $V(\theta)$ is the electrostatic potential at the surface and $V_{0}$ the surface potential of the sphere.

The radius $R_{0}$ of the compound nucleus is defined as:

$$
R_{0}=\left(1.28 A^{1 / 3}-0.76+0.8 A^{-1 / 3}\right) \mathrm{fm}
$$

which leads, for example, to $R_{0}=5.3 \mathrm{fm}$ and $r_{0}=1.15 \mathrm{fm}$ for ${ }^{98} \mathrm{Zr}$ and $R_{0}=7.5 \mathrm{fm}$ and $r_{0}=1.18 \mathrm{fm}$ for ${ }^{255} \mathrm{Fm}$. The radius of the two fragments is calculated assuming volume conservation.

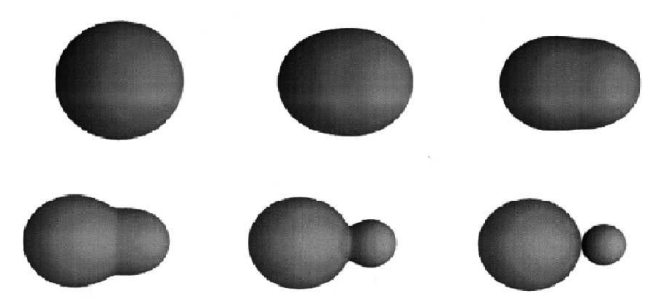

Fig. 1. Selected shape sequence to simulate the one-body shape evolution (for a given asymmetry) 
As in previous works, the one-body shape sequence is described within two joined elliptic lemniscatoids which allow to simulate the development of a deep neck in compact and little elongated shapes with almost spherical ends (see Fig. 1). The shape sequence depends on two parameters: the distance $r$ between the two halves of the system and the ratio $R_{2} / R_{1}$ between the radii of the future fragments. The proximity energy is very important in this deformation path.

\subsection{Two separated ellipsoids}

For two-body shapes, the coaxial ellipsoidal deformations have been considered [26] (see Fig. 2). The system configuration depends on two parameters: the ratios $s_{i}$ $(i=1,2)$ between the transverse semi-axis $a_{i}$ and the radial semi-axis $c_{i}$ of the two different fragments

$$
a_{i}=R_{i} s_{i}^{1 / 3} \quad \text { and } \quad c_{i}=R_{i} s_{i}^{-2 / 3} .
$$
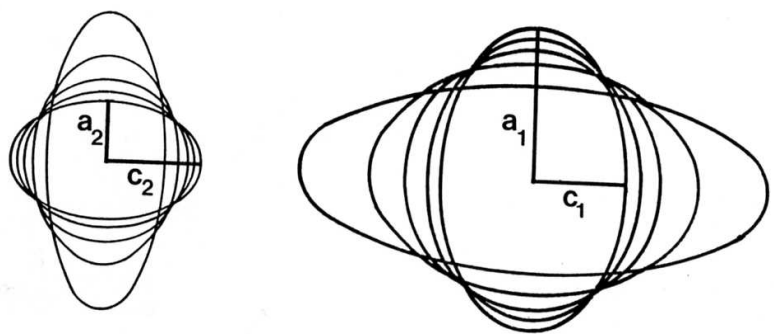

Fig. 2. Two coaxial ellipsoid configuration describing the two-body shape part of the fission barrier. The fission axis is the common axis of revolution

The prolate deformation is characterized by $s \leq 1$ and the related eccentricity is written as $e^{2}=1-s^{2}$ while in the oblate case $s \geq 1$ and $e^{2}=1-s^{-2}$. The volume and surface energies are $E_{V_{12}}=E_{V_{1}}+E_{V_{2}}$ and $E_{S_{12}}=E_{S_{1}}+E_{S_{2}}$. In the prolate case, the relative surface energy reads

$$
B_{S i}=\frac{\left(1-e_{i}^{2}\right)^{1 / 3}}{2}\left[1+\frac{\sin ^{-1}\left(e_{i}\right)}{e_{i}\left(1-e_{i}^{2}\right)^{1 / 2}}\right]
$$

and in the oblate case

$$
B_{S i}=\frac{\left(1+\epsilon_{i}^{2}\right)^{1 / 3}}{2}\left[1+\frac{\ln \left(\epsilon_{i}+\left(1+\epsilon_{i}^{2}\right)^{1 / 2}\right)}{\epsilon_{i}\left(1+\epsilon_{i}^{2}\right)^{1 / 2}}\right], \quad \epsilon_{i}^{2}=s_{i}^{2}-1 .
$$

The Coulomb self-energy of the spheroid $i$ is

$$
E_{C, \text { self }}=\frac{3 e^{2} Z_{i}^{2} B_{c i}}{5 R_{i}}
$$


The relative self-energy is, in the prolate case

$$
B_{C i}=\frac{\left(1-e_{i}^{2}\right)^{1 / 3}}{2 e_{i}} \ln \frac{1+e_{i}}{1-e_{i}}
$$

and, in the oblate case

$$
B_{C i}=\frac{\left(1+\epsilon_{i}^{2}\right)^{1 / 3}}{\epsilon_{i}} \tan ^{-1} \epsilon_{i} .
$$

The Coulomb interaction energy between the two fragments reads

$$
E_{C, \text { int }}=\frac{e^{2} Z_{1} Z_{2}}{r}\left[s\left(\lambda_{1}\right)+s\left(\lambda_{2}\right)-1+S\left(\lambda_{1}, \lambda_{2}\right)\right], \quad \lambda_{i}^{2}=\frac{c_{i}^{2}-a_{i}^{2}}{r^{2}},
$$

$r$ being, as before, the distance between the two mass centres. In the prolate case, $s\left(\lambda_{i}\right)$ is expressed as

$$
s\left(\lambda_{i}\right)=\frac{3}{4}\left(\frac{1}{\lambda_{i}}-\frac{1}{\lambda_{i}^{3}}\right) \ln \left(\frac{1+\lambda_{i}}{1-\lambda_{i}}\right)+\frac{3}{2 \lambda_{i}^{2}},
$$

while, for the oblate shapes,

$$
s\left(\lambda_{i}\right)=\frac{3}{2}\left(\frac{1}{\omega_{i}}+\frac{1}{\omega_{i}^{3}}\right) \tan ^{-1} \omega_{i}-\frac{3}{2 \omega_{i}^{2}}, \quad \omega_{i}^{2}=-\lambda_{i}^{2} .
$$

$S\left(\lambda_{1}, \lambda_{2}\right)$ can be represented in the form of a two-fold summation

$$
S\left(\lambda_{1}, \lambda_{2}\right)=\sum_{j=1}^{\infty} \sum_{k=1}^{\infty} \frac{3}{(2 j+1)(2 j+3)} \frac{3}{(2 k+1)(2 k+3)} \frac{(2 j+2 k) !}{(2 j) !(2 k) !} \lambda_{1}^{2 j} \lambda_{2}^{2 k} .
$$

\section{Shell Energy}

The shape-dependent shell corrections have been determined within the Droplet Model expressions [11] which allow to reproduce accurately the nuclear masses and fission barriers

$$
E_{\text {shell }}=E_{\text {shell }}^{\text {sphere }}\left(1-2.6 \alpha^{2}\right) e^{-\alpha^{2}} .
$$

The shell corrections for a spherical nucleus are

$$
E_{\text {shell }}^{\text {sphere }}=5.8\left[(F(N)+F(Z)) /(0.5 A)^{2 / 3}-0.26 A^{1 / 3}\right] \mathrm{MeV},
$$

where, for $M_{i-1}<X<M_{i}$,

$$
F(X)=q_{i}\left(X-M_{i-1}\right)-0.6\left(X^{5 / 3}-M_{i-1}^{5 / 3}\right) .
$$

$M_{i}$ are the magic numbers and

$$
q_{i}=0.6\left(M_{i}^{5 / 3}-M_{i-1}^{5 / 3}\right) /\left(M_{i}-M_{i-1}\right) .
$$


The selected highest proton magic number is 114 while, for the two highest neutron magic numbers, the values 126 and 184 have been retained

$$
\alpha^{2}=(\delta R)^{2} / a^{2} .
$$

The distortion $\alpha a$ measures the deviation of the nuclear surface from the sphere, a quantity which incorporates indiscriminately all types of deformation. The range $a$ has been chosen to be $0.34 r_{0}$. For the two-body shapes, the total shell energy is the sum of the shell corrections for each deformed fragment.

\section{Pairing Energy}

The pairing energy has been calculated with the following expressions used in a recent version of the Thomas-Fermi model [12].

For odd $Z$, odd $N$ and $N=Z$ nuclei:

$$
E_{\text {Pairing }}=4.8 / N^{1 / 3}+4.8 / Z^{1 / 3}-6.6 / A^{2 / 3}+30 / A .
$$

For odd $Z$, odd $N$ and $N \neq Z$ nuclei:

$$
E_{\text {Pairing }}=4.8 / N^{1 / 3}+4.8 / Z^{1 / 3}-6.6 / A^{2 / 3} .
$$

For odd $Z$, even $N$ nuclei:

$$
E_{\text {Pairing }}=4.8 / Z^{1 / 3}
$$

For even $Z$, odd $N$ nuclei:

$$
E_{\text {Pairing }}=4.8 / N^{1 / 3} \text {. }
$$

For even $Z$, even $N$ nuclei:

$$
E_{\text {Pairing }}=0 .
$$

\section{Potential Barriers}

The dependence of the deformation energy on the shape sequence and introduction of the microscopic corrections is displayed in Fig. 3 for an asymmetric fission path of the ${ }^{230} \mathrm{Th}$ nucleus. The shell effects generate the slightly deformed ground state and contribute to the formation of the first peak. The proximity energy flattens the potential energy curve and will explain with the shell effects the formation of a deep second minimum lodging the superdeformed isomeric states for heaviest nuclei. In the exit channel corresponding to the two-sphere approximation the top of the barrier $(r=12.6 \mathrm{fm}$ on this example) is reached after the rupture of the matter bridge between the two spherical fragments $(r=11.4 \mathrm{fm})$. Then, the top corresponds to two separated spherical fragments maintained in unstable equilibrium by the balance between the attractive nuclear forces and the repulsive Coulomb ones. In this path and mass range, the introduction of the shell and pairing effects for two-sphere 


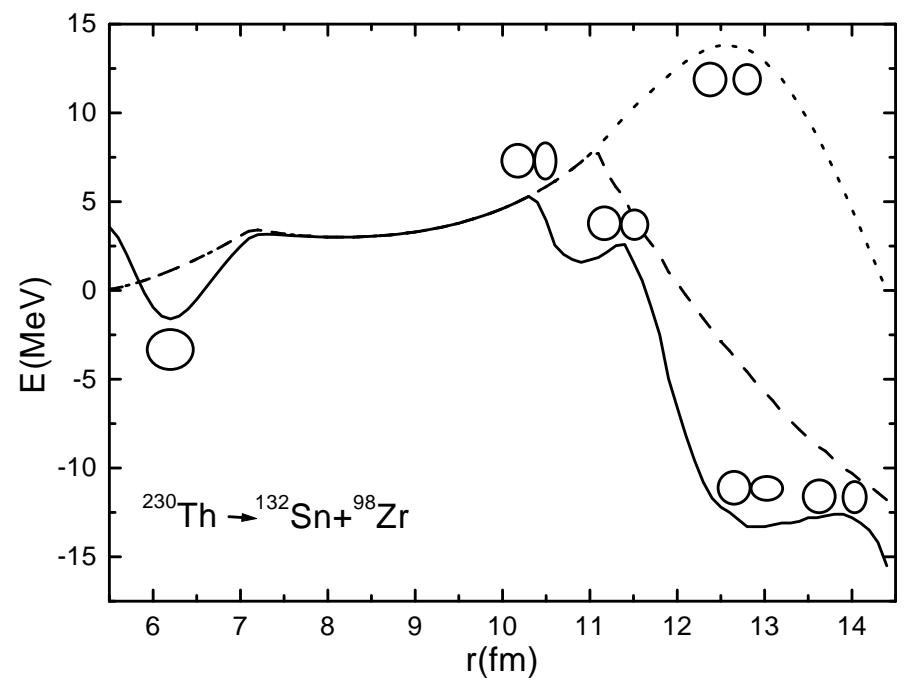

Fig. 3. Asymmetric fission barrier of a ${ }^{230} \mathrm{Th}$ nucleus emitting a doubly magic nucleus ${ }^{132} \mathrm{Sn}$. The dotted and dashed curves give respectively the macroscopic energy within the two-sphere approximation and the ellipsoidal deformations for the two-body shapes. The solid line includes the shell and pairing energies. $r$ is the distance between mass centres

shapes is not sufficient to reproduce accurately the experimental data on the fission barrier heights of actinide nuclei. When the ellipsoidal deformations of the fragments are taken into account the transition between one-body and two-body shapes corresponds to the passage (at $r=11 \mathrm{fm}$ for ${ }^{230} \mathrm{Th}$ ) from a one-body shape with spherical ends and a deep neck to two touching ellipsoidal fragments, one or both of them being slightly oblate. The barrier height is reduced by several MeV. The introduction of the shell effects still lowers the second peak and shifts it to an inner position ( $r=10.3 \mathrm{fm}$ here). It even leads to a third minimum and third peak in this asymmetric decay path. A plateau appears also at larger distances around $10 \mathrm{MeV}$ below the ground state. It is due to the persistence of the prolate deformation of the lightest fragment. The end of the plateau corresponds to the end of the contact between the two fragments and to a rapid transition from prolate to oblate shapes for the non-magical fragment and the vanishing of the proximity energy. Later on, this second fragment returns to a prolate shape when the interaction Coulomb energy is smaller.

The potential barriers for the ${ }^{232,235,238} \mathrm{U},{ }^{238,240,243} \mathrm{Pu},{ }^{243,244} \mathrm{Am},{ }^{243} \mathrm{Cm}$, ${ }^{250} \mathrm{Bk}$ and ${ }^{250} \mathrm{Cf}$ nuclei are shown in Figs. 4 to 7 . It is important to mention that to obtain these barriers the only input parameters are $A_{1}, Z_{1}, A_{2}$ and $Z_{2}$ and that the calculations are rapid and, consequently, can be integrated in a more complex code devoted to fragment distributions and fission cross sections. 

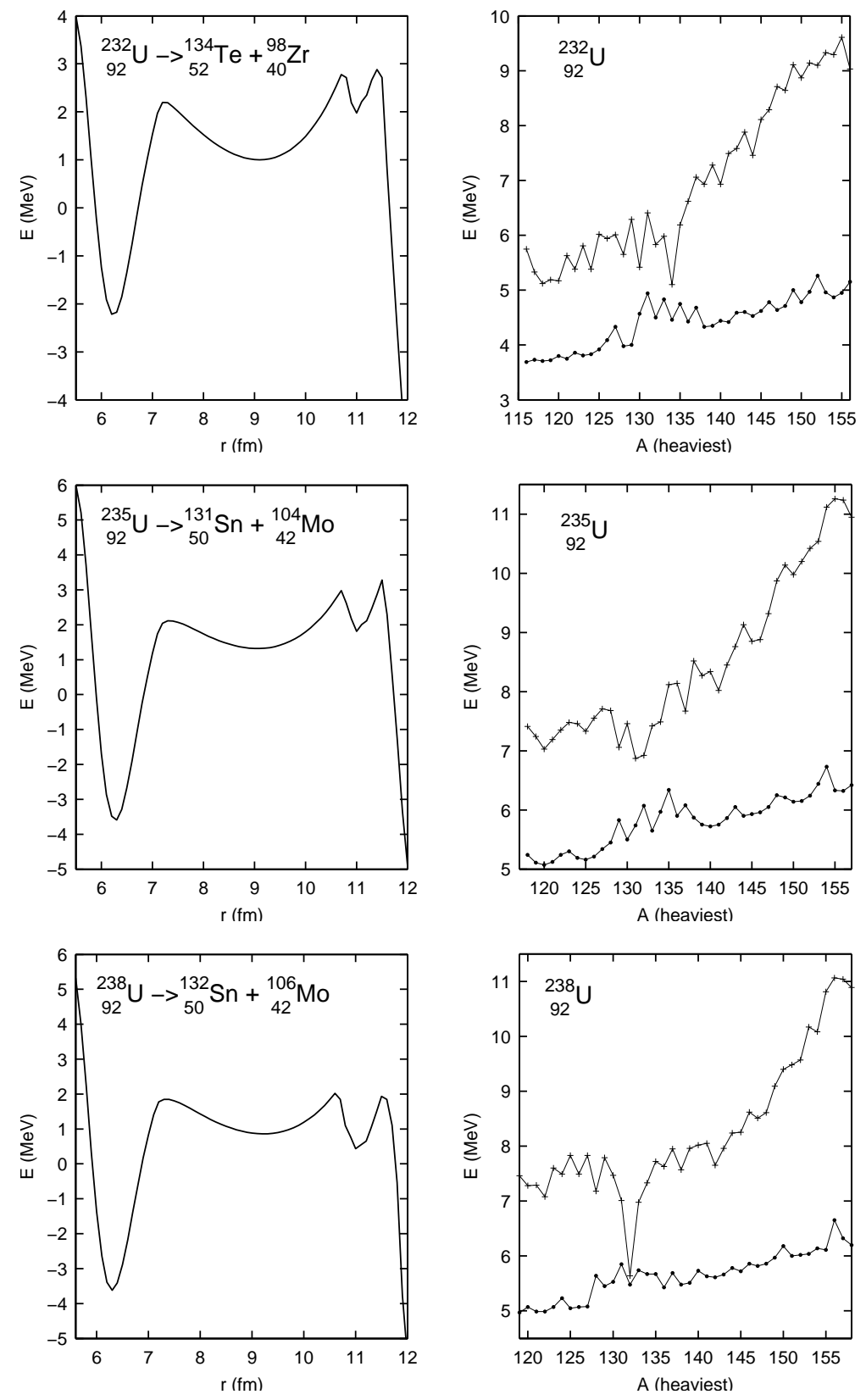

Fig. 4. On the left, multiple-humped fission barriers in the mentioned asymmetric fission path for ${ }^{232,235,238} \mathrm{U}$. On the right, inner (full circles) and outer (crosses) fission barrier heights as a function of the mass of the heaviest fragment 

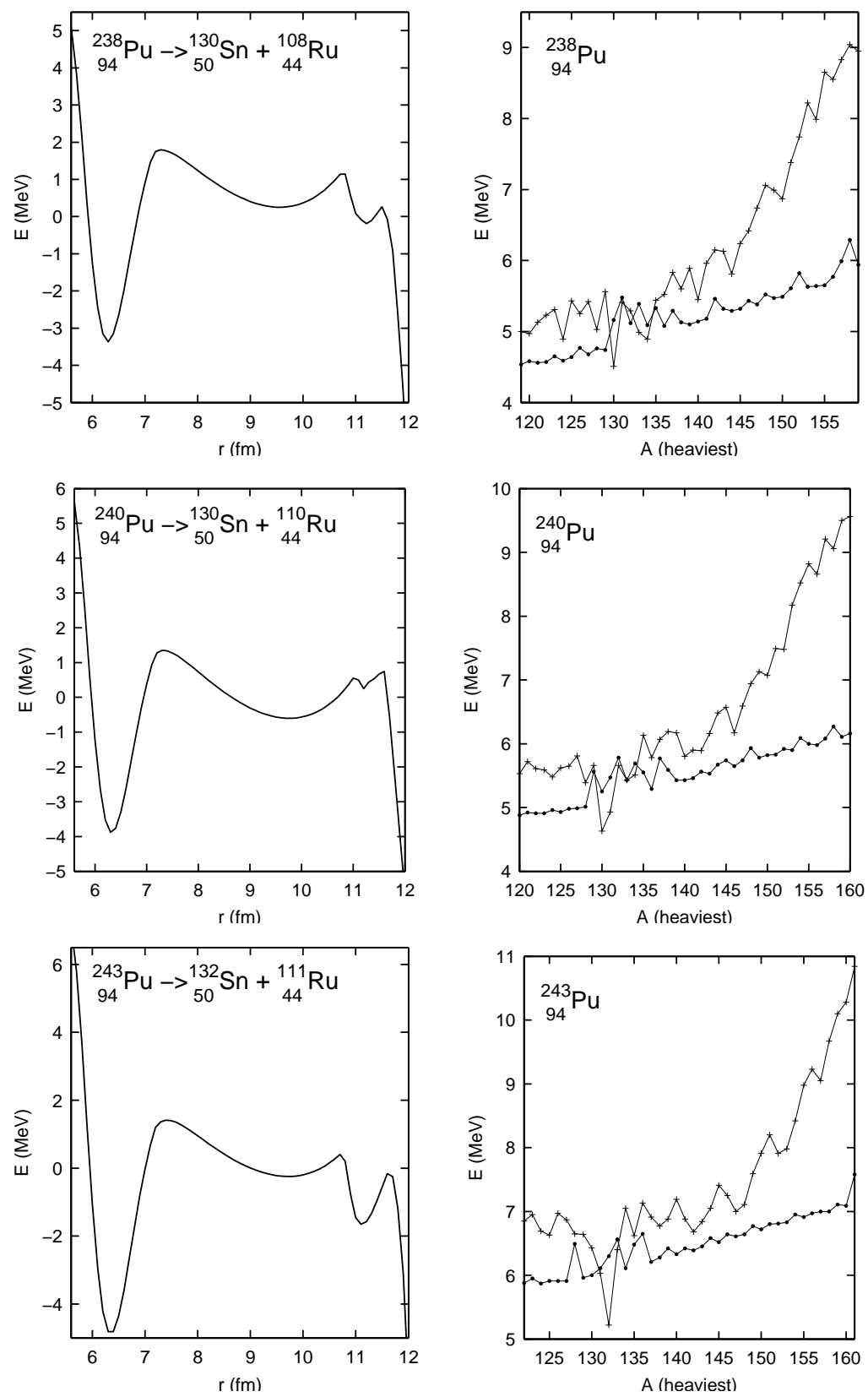

Fig. 5. On the left, multiple-humped fission barriers in the mentioned asymmetric fission path for ${ }^{238,240,243} \mathrm{Pu}$. On the right, inner (full circles) and outer (crosses) fission barrier heights as a function of the mass of the heaviest fragment 

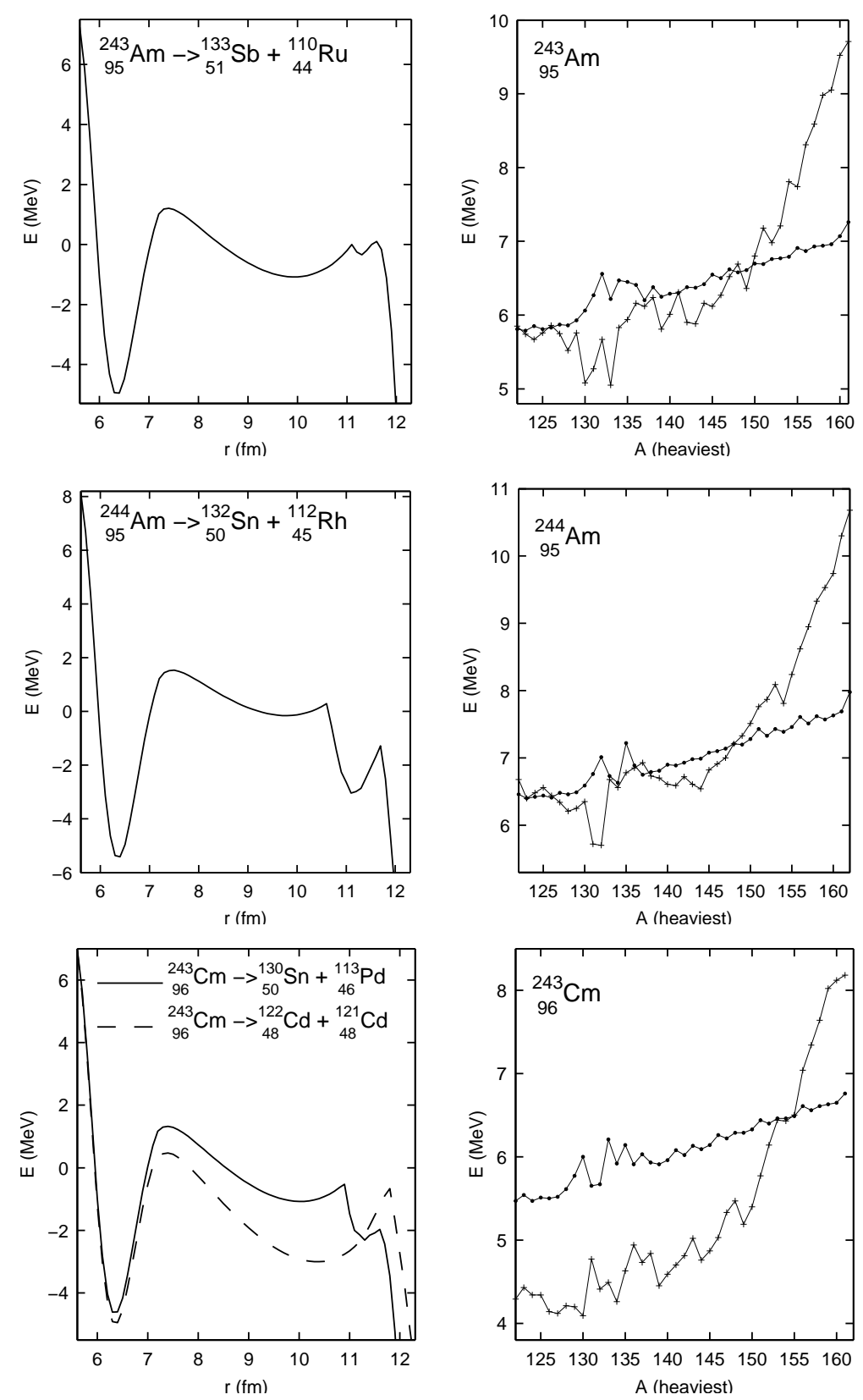

Fig. 6. Fission barriers for ${ }^{243,244} \mathrm{Am}$ and ${ }^{243} \mathrm{Cm}$. The inner and outer fission barrier heights are given, on the right, respectively by the full circles and crosses 

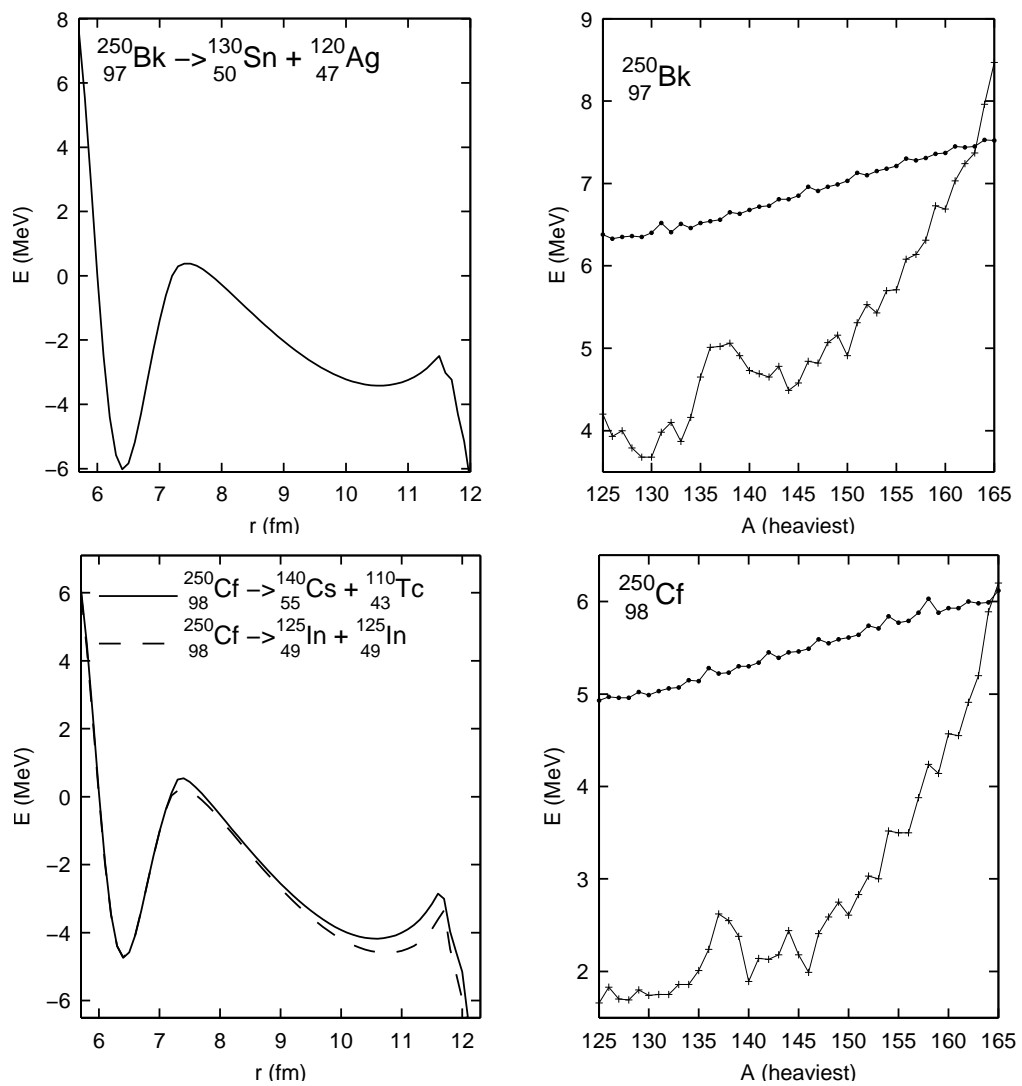

Fig. 7. Fission barriers for ${ }^{250} \mathrm{Bk}$ and ${ }^{250} \mathrm{Cf}$. The inner and outer fission barrier heights are given, on the right, respectively by the full circles and crosses

For a given mass asymmetry, the charge asymmetry which minimizes the deformation energy has been selected. The proximity energy and the attenuated microscopic effects are responsible for the formation of a second one-body shape minimum. The heights of the two peaks generally increase with the asymmetry but the shell and pairing corrections induce strong variations from this global behaviour. Their main effect is to favour, for the $\mathrm{U}, \mathrm{Pu}, \mathrm{Am}$ and $\mathrm{Cm}$ isotopes, an asymmetric path where one fragment is close to the doubly magic number ${ }_{50}^{132} \mathrm{Sn}$ nucleus, and, consequently, keeps an almost spherical shape. This effect is less pronounced for ${ }^{243} \mathrm{Cm}$ and ${ }^{250} \mathrm{Cf}$ since for nuclei with $Z \sim 100$ the symmetric fission gives fragments with a charge of around 50. For these nuclei a symmetric and an asymmetric exit channels are compared on the lower part of Figs. 6 and 7. A third minimum and third peak appear in the asymmetric decay path. There is no third barrier in the symmetric deformation path. The origin of the third peak is investigated in the next section. 
Table 1. Comparison between theoretical (t) and experimental (e) $[3,5,16,17]$ barrier characteristics for actinide nuclei. $E_{a}, E_{b}$ and $E_{c}$ are the first, second and third peak heights while $E_{2}$ and $E_{3}$ are the energies of the second and third potential minima relatively to the ground state energy (in $\mathrm{MeV}$ ).

\begin{tabular}{|c|c|c|c|c|c|c|c|c|c|}
\hline & Reaction & $E_{a}(t)$ & $E_{a}(e)$ & $E_{2}(t)$ & $E_{2}(e)$ & $E_{b}(t)$ & $E_{b}(e)$ & $E_{3}(t)$ & $E_{c}(t)$ \\
\hline${ }_{90}^{230} \mathrm{Th}$ & $\rightarrow{ }_{50}^{132} \mathrm{Sn}+{ }_{40}^{98} \mathrm{Zr}$ & 4.8 & - & 4.6 & - & 6.9 & - & 3.2 & 4.2 \\
\hline${ }_{90}^{231} \mathrm{Th}$ & $\rightarrow{ }_{50}^{132} \mathrm{Sn}+{ }_{40}^{99} \mathrm{Zr}$ & 5.5 & $\begin{array}{l}- \\
-\end{array}$ & 5.2 & - & 7.1 & 6.5 & $\begin{array}{l}3.9 \\
\mathrm{e}: 5.6\end{array}$ & $\begin{array}{l}6.9 \\
\text { e: } 6.3\end{array}$ \\
\hline${ }_{90}^{233} \mathrm{Th}$ & $\rightarrow{ }_{50}^{132} \mathrm{Sn}+{ }_{40}^{101} \mathrm{Zr}$ & 5.6 & $\begin{array}{l}- \\
-\end{array}$ & 5.15 & - & 7.0 & 6.8 & $\begin{array}{l}5.0 \\
\text { e: } 5.2\end{array}$ & $\begin{array}{l}7.8 \\
\text { e: } 6.8\end{array}$ \\
\hline${ }_{92}^{232} \mathrm{U}$ & $\rightarrow{ }_{52}^{134} \mathrm{Te}+{ }_{40}^{98} \mathrm{Zr}$ & 4.5 & 4.9 & 3.2 & - & 5.0 & 5.4 & 4.2 & 5.1 \\
\hline${ }_{92}^{234} \mathrm{U}$ & $\rightarrow{ }_{50}^{131} \mathrm{Sn}+{ }_{42}^{103} \mathrm{Mo}$ & 5.0 & $\begin{array}{c}5.6 \\
-\end{array}$ & 4.4 & - & 5.9 & 5.5 & $\begin{array}{l}3.7 \\
\mathrm{e}: 3.1\end{array}$ & 5.6 \\
\hline${ }_{92}^{235} \mathrm{U}$ & $\rightarrow{ }_{50}^{131} \mathrm{Sn}+{ }_{42}^{104} \mathrm{Mo}$ & 5.7 & 5.7 & 4.9 & 2.5 & 6.6 & 5.8 & 5.4 & 6.9 \\
\hline${ }_{92}^{236} \mathrm{U}$ & $\rightarrow{ }_{50}^{132} \mathrm{Sn}+{ }_{42}^{104} \mathrm{Mo}$ & 5.5 & $\begin{array}{c}5.6 \\
-\end{array}$ & 4.8 & 2.3 & 6.2 & 5.5 & $\begin{array}{l}3.1 \\
\text { e: } 3.15\end{array}$ & 4.4 \\
\hline${ }_{92}^{237} \mathrm{U}$ & $\rightarrow{ }_{50}^{132} \mathrm{Sn}+{ }_{42}^{105} \mathrm{Mo}$ & 6.1 & 6.1 & 5.3 & 2.5 & 6.5 & 5.9 & 3.6 & 6.2 \\
\hline${ }_{92}^{238} \mathrm{U}$ & $\rightarrow{ }_{50}^{132} \mathrm{Sn}+{ }_{42}^{106} \mathrm{Mo}$ & 5.5 & 5.7 & 4.5 & 2.6 & 5.6 & 5.7 & 4.1 & 5.6 \\
\hline${ }_{92}^{239} \mathrm{U}$ & $\rightarrow{ }_{50}^{132} \mathrm{Sn}+{ }_{42}^{107} \mathrm{Mo}$ & 6.1 & 5.8 & 5.0 & 1.9 & 6.0 & 6.0 & 4.6 & 7.0 \\
\hline${ }_{93}^{238} \mathrm{~Np}$ & $\rightarrow{ }_{50}^{132} \mathrm{Sn}+{ }_{43}^{106} \mathrm{Tc}$ & 6.9 & 6.0 & 6.2 & 2.3 & 7.1 & 6.0 & 2.5 & 5.1 \\
\hline${ }_{94}^{238} \mathrm{Pu}$ & $\rightarrow{ }_{50}^{130} \mathrm{Sn}+{ }_{44}^{108} \mathrm{Ru}$ & 5.2 & 5.6 & 3.6 & 2.7 & 4.5 & 5.0 & 3.2 & 3.6 \\
\hline${ }_{94}^{239} \mathrm{Pu}$ & $\rightarrow{ }_{50}^{130} \mathrm{Sn}+{ }_{44}^{109} \mathrm{Ru}$ & 5.8 & 6.2 & 4.1 & 2.6 & 5.0 & 5.5 & 4.1 & 5.6 \\
\hline${ }_{94}^{240} \mathrm{Pu}$ & $\rightarrow{ }_{50}^{130} \mathrm{Sn}+{ }_{44}^{110} \mathrm{Ru}$ & 5.3 & 5.7 & 3.3 & 2.4 & 4.6 & 5.1 & & \\
\hline${ }_{94}^{241} \mathrm{Pu}$ & $\rightarrow{ }_{50}^{131} \mathrm{Sn}+{ }_{44}^{110} \mathrm{Ru}$ & 6.1 & 6.0 & 4.4 & 1.9 & 5.65 & 5.5 & 5.1 & 5.5 \\
\hline${ }_{94}^{243} \mathrm{Pu}$ & $\rightarrow{ }_{50}^{132} \mathrm{Sn}+{ }_{44}^{111} \mathrm{Ru}$ & 6.3 & 5.9 & 4.6 & 1.7 & 5.2 & 5.4 & 3.2 & 4.6 \\
\hline${ }_{95}^{242} \mathrm{Am}$ & $\rightarrow{ }_{50}^{131} \mathrm{Sn}+{ }_{45}^{111} \mathrm{Rh}$ & 6.8 & 6.5 & 5.1 & 2.9 & 5.7 & 5.4 & 4.1 & 5.1 \\
\hline${ }_{95}^{243} \mathrm{Am}$ & $\mathrm{n} \rightarrow{ }_{51}^{133} \mathrm{Sn}+{ }_{44}^{110} \mathrm{Ru}$ & 6.2 & 5.9 & 6.4 & 2.3 & 5.0 & 5.4 & 4.7 & 5.1 \\
\hline${ }_{95}^{244} \mathrm{Am}$ & $\rightarrow{ }_{50}^{132} \mathrm{Sn}+{ }_{45}^{112} \mathrm{Rh}$ & 7.0 & 6.3 & 5.3 & 2.8 & 5.7 & 5.4 & 2.4 & 4.2 \\
\hline${ }_{96}^{243} \mathrm{Cm}$ & $\rightarrow{ }_{50}^{130} \mathrm{Sn}+{ }_{46}^{113} \mathrm{Pd}$ & 6.0 & 6.4 & 3.6 & 1.9 & 4.2 & 4.2 & 2.4 & 2.7 \\
\hline${ }_{96}^{243} \mathrm{Cm}$ & $\rightarrow{ }_{48}^{122} \mathrm{Cd}+{ }_{48}^{121} \mathrm{Cd}$ & 5.5 & 6.4 & 2.0 & 1.9 & 4.3 & 4.2 & & \\
\hline${ }_{96}^{245} \mathrm{Cm}$ & $1 \rightarrow{ }_{50}^{130} \mathrm{Sn}+{ }_{46}^{115} \mathrm{Pd}$ & 6.0 & 6.2 & 3.1 & 2.1 & 3.7 & 4.8 & & \\
\hline${ }_{96}^{248} \mathrm{Cm}$ & $\rightarrow{ }_{50}^{130} \mathrm{Sn}+{ }_{46}^{118} \mathrm{Pd}$ & 5.3 & 5.7 & 2.0 & - & 3.0 & 4.6 & & \\
\hline${ }_{97}^{250} \mathrm{Bk}$ & $\rightarrow{ }_{50}^{130} \mathrm{Sn}+{ }_{47}^{120} \mathrm{Ag}$ & 6.4 & 6.1 & 2.6 & - & 3.7 & 4.1 & & \\
\hline${ }_{98}^{250} \mathrm{Cf}$ & $\rightarrow{ }_{49}^{125} \mathrm{In}+{ }_{49}^{125} \mathrm{In}$ & 4.9 & 5.6 & 0.1 & - & 1.7 & - & & \\
\hline${ }_{98}^{250} \mathrm{Cf}$ & $\rightarrow{ }_{55}^{140} \mathrm{Cs}+{ }_{43}^{110} \mathrm{Tc}$ & 5.3 & 5.6 & 0.5 & - & 1.9 & - & & \\
\hline${ }_{99}^{256} \mathrm{Es}$ & $\rightarrow{ }_{50}^{128} \mathrm{Sn}+{ }_{49}^{128} \mathrm{In}$ & 5.9 & 4.8 & 0.8 & - & 2.4 & - & & \\
\hline $\begin{array}{l}255 \\
100\end{array}$ & $\rightarrow{ }_{51}^{127} \mathrm{Sb}+{ }_{49}^{128} \mathrm{In}$ & 5.5 & 5.7 & 0.3 & - & 1.9 & - & & \\
\hline
\end{tabular}

The calculated and experimental energies of the maxima and minima of the fission barriers are compared in Table 1. The choice of the most probable fission path is difficult for some isotopes since there is a true degenerescence in energy between several paths of the multi-dimensional potential surfaces, particularly for 
the heaviest elements where the symmetric path seems more probable. There is a very good agreement between the experimental and theoretical heights $E_{a}$ and $E_{b}$ of the two peaks. The predicted value of the second minimum energy is a little too high. The still sparse but exciting data for the third barrier are correctly reproduced. For the heaviest nuclei the external barrier disappears since the attractive proximity forces can no more compensate for the repulsive Coulomb forces.

\section{Third Barrier}

The origin of the existence of the third well in the asymmetric decay path is examined now (see Fig. 8). The dashed line represents the potential for two touching ellipsoids when the one-body shape is still energetically favoured. The second peak (but first on the figure) corresponds to the point where these touching ellipsoids begin to give the lowest energy. The heaviest fragment is a magic nucleus. It therefore preserves its almost spherical shape. The non-magic fragment was born in an oblate shape $(s \sim 1.4)$, due to the small distance between the mass centres at this step. When this distance increases, the ratio s decreases, because of the proximity energy which tends to keep close the two tips of the fragments. Thus, the lightest fragment remaining in contact with the other spherical fragment approaches the spherical shape and its shell energy increases to reach a maximum which is at the origin of the third peak and which corresponds to two touching different spheres. Before reaching this third peak a third minimum appears. Its shape is hyperdeformed and asymmetric in agreement with the experimental data [3]. Later on, the proximity forces maintain the two fragments in contact and the shape of the smallest one
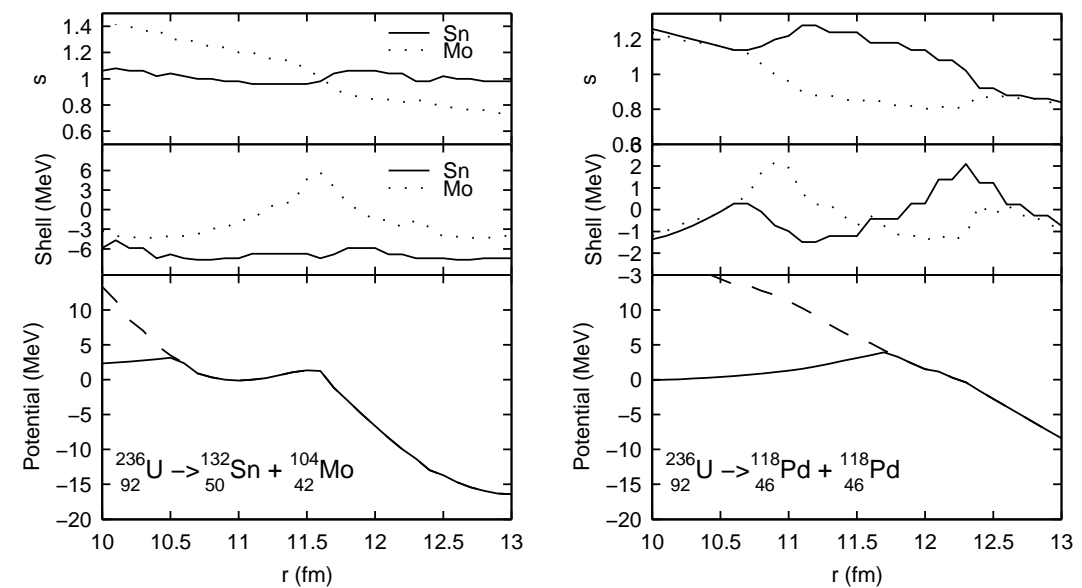

Fig. 8. Fission barriers, shell energies and ratio of the semi-axes of the two ellipsoidal fragments for an asymmetric decay channel and the symmetric one for ${ }^{236} \mathrm{U}$. On the lowest part, the fission barrier is given by the solid line 

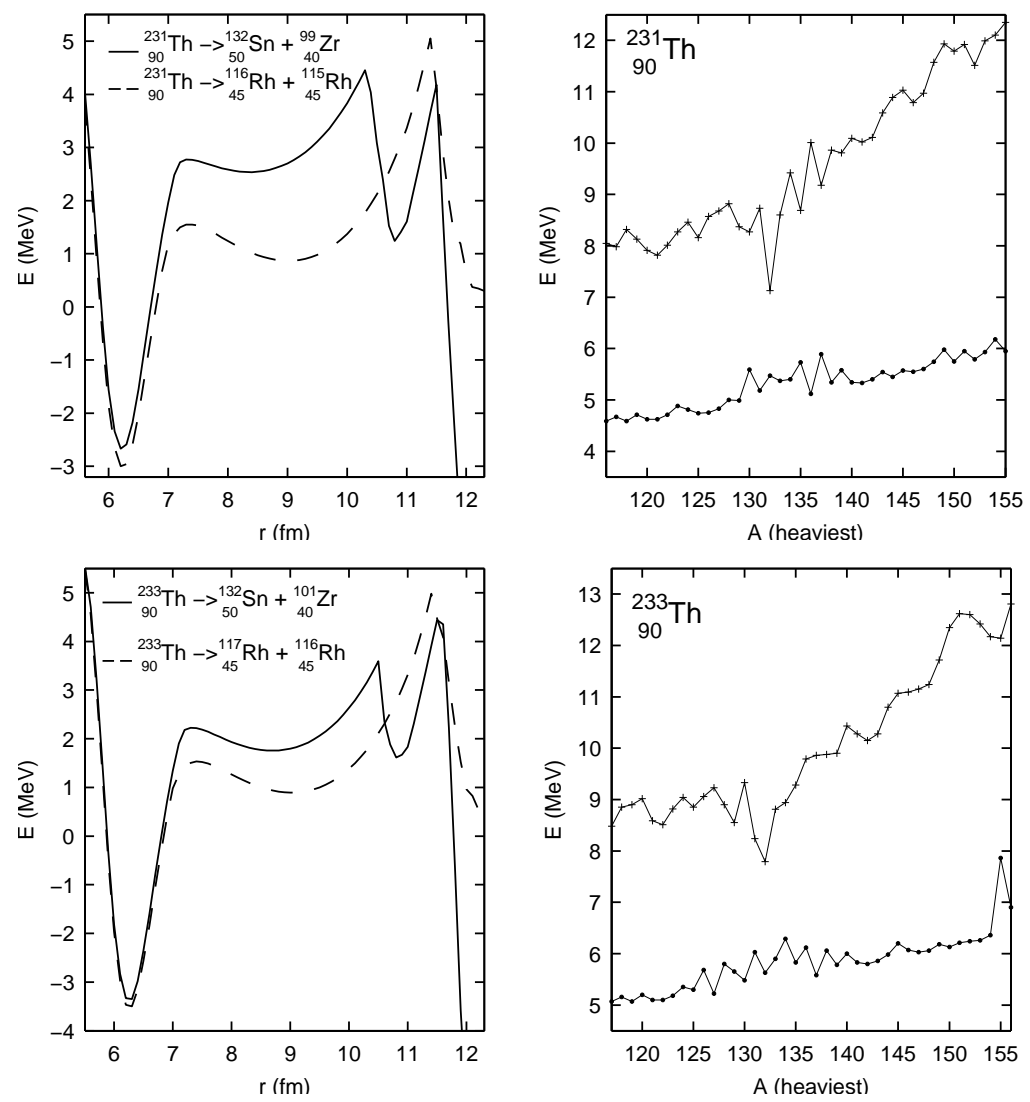

Fig. 9. Fission barriers for ${ }^{231,233}$ Th and two different decay channels. The inner and outer fission barrier heights are given, on the right, respectively by the full circles and crosses

evolves to prolate shapes $(s<1)$ and the shell corrections decrease. The third barrier appears only in the asymmetric decay path and for some specific nuclei. In the symmetric mass exit path, the proximity and Coulomb energies counterbalance the smallest shell effects and induce an asymmetric shape, the two fragments remain in contact but one fragment is oblate while the other one is prolate. With increasing distance between the mass centres the two nuclei become prolate.

The dependence of the fission barrier heights and profiles on the asymmetry for the ${ }^{231,233} \mathrm{Th}$ and ${ }^{234,236} \mathrm{U}$ nuclei, for which experimental data on the third barrier exist, are given in Figs. 9 and 10. The position of the second peak in the symmetric decay path corresponds to the position of the third peak in the asymmetric deformation path. Clearly the magicity of some $\mathrm{Sn}$ isotopes plays the main role. 

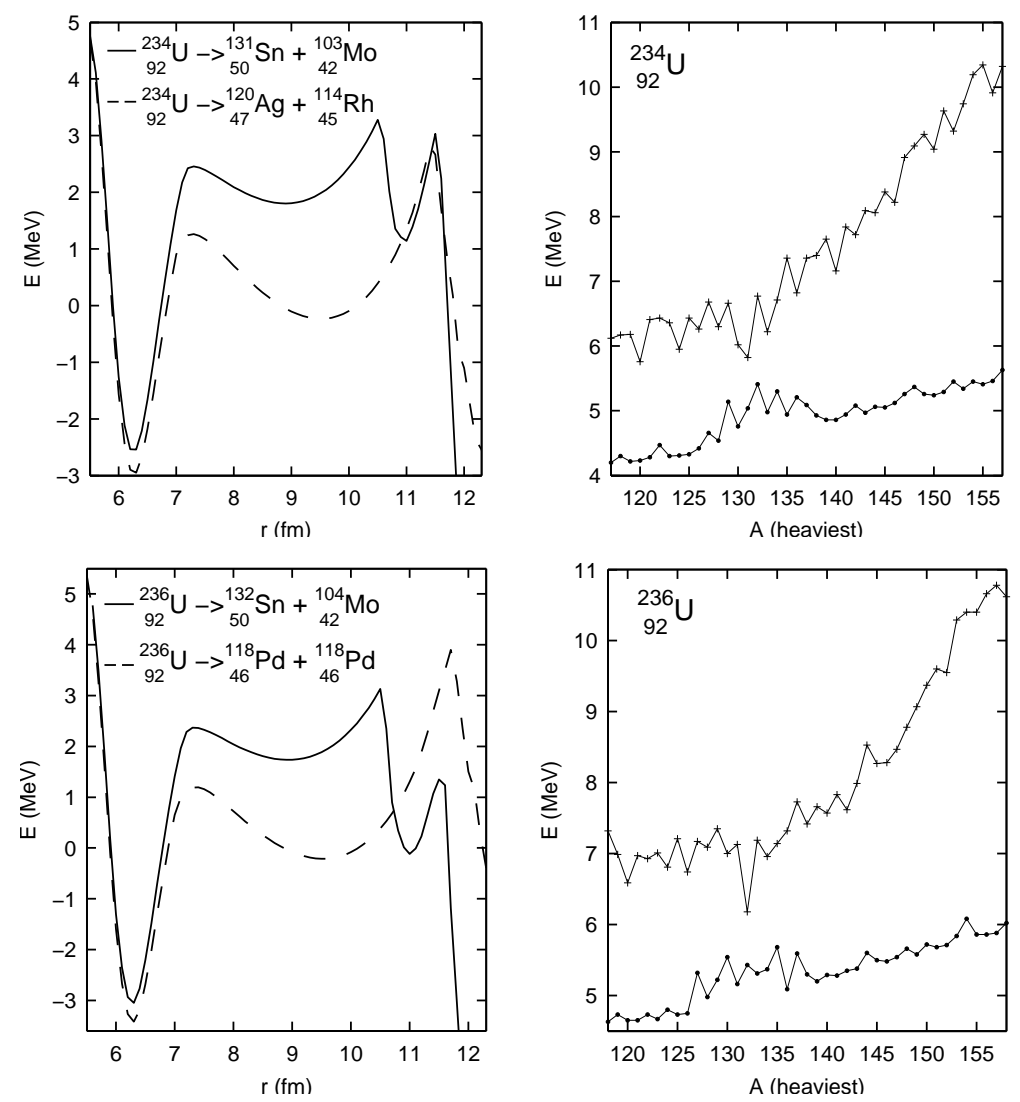

Fig. 10. Fission barriers for ${ }^{234,236} \mathrm{U}$. The inner and outer fission barrier heights are given, on the right, respectively by the full circles and crosses

\section{Half-Lives}

Within this asymmetric fission model the decay constant is simply given by $\lambda=\nu_{0} P$. The assault frequency $\nu_{0}$ has been taken as $\nu_{0}=10^{20} \mathrm{~s}^{-1}$. The barrier penetrability $P$ is calculated within the action integral

$$
P=\exp \left[-\frac{2}{\hbar} \int_{r_{\text {in }}}^{r_{\text {out }}} \sqrt{2 B(r)\left(E(r)-E_{\text {g.s. }}\right)} d r\right] .
$$

The inertia $B(r)$ is related to the reduced mass by

$$
B(r)=\mu\left[1+24 \exp \left(-3.25\left(r-R_{\mathrm{sph}}\right) / R_{0}\right)\right],
$$

where $R_{\mathrm{sph}}$ is the distance between the mass centres of the future fragments in the initial sphere, $R_{\mathrm{sph}} / R_{0}=0.75$ in the symmetric case. 
For shapes near the ground state the inertia is largely above the irrotational flow value since a large amount of internal reorganization occurs at level crossings. For highly deformed shapes the reduced mass is reached asymptotically. The partial half-life is finally obtained by $T_{1 / 2}=(\ln 2) / \lambda$.

Table 2. Comparison between experimental [17] and theoretical spontaneous fission half-lives of actinide nuclei

\begin{tabular}{llc}
\hline \multicolumn{1}{c}{ Reaction } & $T_{1 / 2, \exp (s)}$ & $T_{1 / 2, \text { theo }(s)}$ \\
\hline${ }_{92}^{232} \mathrm{U} \rightarrow{ }_{52}^{134} \mathrm{Te}+{ }_{40}^{98} \mathrm{Zr}$ & $2.5 \times 10^{21}$ & $3.6 \times 10^{16}$ \\
${ }_{92}^{234} \mathrm{U} \rightarrow{ }_{50}^{131} \mathrm{Sn}+{ }_{42}^{103} \mathrm{Mo}$ & $4.7 \times 10^{23}$ & $8 \times 10^{19}$ \\
${ }_{92}^{235} \mathrm{U} \rightarrow{ }_{50}^{131} \mathrm{Sn}+{ }_{42}^{104} \mathrm{Mo}$ & $3.1 \times 10^{26}$ & $7.7 \times 10^{23}$ \\
${ }_{92}^{236} \mathrm{U} \rightarrow{ }_{50}^{132} \mathrm{Sn}+{ }_{42}^{104} \mathrm{Mo}$ & $7.8 \times 10^{23}$ & $1.0 \times 10^{22}$ \\
${ }_{92}^{238} \mathrm{U} \rightarrow{ }_{50}^{132} \mathrm{Sn}+{ }_{42}^{106} \mathrm{Mo}$ & $2.6 \times 10^{23}$ & $5.3 \times 10^{22}$ \\
${ }_{94}^{238} \mathrm{Pu} \rightarrow{ }_{50}^{130} \mathrm{Sn}+{ }_{44}^{108} \mathrm{Ru}$ & $1.5 \times 10^{18}$ & $2.6 \times 10^{19}$ \\
${ }_{94}^{239} \mathrm{Pu} \rightarrow{ }_{50}^{130} \mathrm{Sn}+{ }_{44}^{109} \mathrm{Ru}$ & $2.5 \times 10^{23}$ & $4.8 \times 10^{22}$ \\
${ }_{94}^{240} \mathrm{Pu} \rightarrow{ }_{50}^{130} \mathrm{Sn}+{ }_{44}^{110} \mathrm{Ru}$ & $3.7 \times 10^{18}$ & $4.8 \times 10^{19}$ \\
${ }_{95}^{443} \mathrm{Am} \rightarrow{ }_{51}^{133} \mathrm{Sb}+{ }_{44}^{110} \mathrm{Ru}$ & $6.3 \times 10^{21}$ & $1.1 \times 10^{23}$ \\
${ }_{96}^{243} \mathrm{Cm} \rightarrow{ }_{50}^{130} \mathrm{Sn}+{ }_{46}^{113} \mathrm{Pd}$ & $1.7 \times 10^{19}$ & $3 \times 10^{21}$ \\
${ }_{96}^{243} \mathrm{Cm} \rightarrow{ }_{48}^{122} \mathrm{Cd}+{ }_{48}^{121} \mathrm{Cd}$ & $1.7 \times 10^{19}$ & $1.6 \times 10^{18}$ \\
${ }_{96}^{245} \mathrm{Cm} \rightarrow{ }_{50}^{130} \mathrm{Sn}+{ }_{46}^{115} \mathrm{Pd}$ & $4.4 \times 10^{19}$ & $3 \times 10^{20}$ \\
${ }_{96}^{248} \mathrm{Cm} \rightarrow{ }_{50}^{130} \mathrm{Sn}+{ }_{46}^{118} \mathrm{Pd}$ & $1.3 \times 10^{14}$ & $7.7 \times 10^{15}$ \\
${ }_{98}^{250} \mathrm{Cf} \rightarrow{ }_{49}^{125} \mathrm{In}+{ }_{49}^{125} \mathrm{In}$ & $5.2 \times 10^{11}$ & $1.9 \times 10^{9}$ \\
${ }_{98}^{250} \mathrm{Cf} \rightarrow{ }_{52}^{132} \mathrm{Te}+{ }_{46}^{118} \mathrm{Pd}$ & $5.2 \times 10^{11}$ & $1.2 \times 10^{10}$ \\
${ }_{98}^{250} \mathrm{Cf} \rightarrow{ }_{55}^{140} \mathrm{Cs}+{ }_{43}^{110} \mathrm{Tc}$ & $5.2 \times 10^{11}$ & $4.9 \times 10^{11}$ \\
${ }_{99}^{255} \mathrm{Es} \rightarrow{ }_{50}^{128} \mathrm{Sn}+{ }_{49}^{127} \mathrm{In}$ & $8.4 \times 10^{10}$ & $8 \times 10^{9}$ \\
${ }_{100}^{256} \mathrm{Fm} \rightarrow{ }_{50}^{128} \mathrm{Sn}+{ }_{50}^{1128} \mathrm{Sn}$ & $1.0 \times 10^{4}$ & 45 \\
${ }_{100}^{256} \mathrm{Fm} \rightarrow{ }_{47}^{121} \mathrm{Ag}+{ }_{53}^{135} \mathrm{I}$ & $1.0 \times 10^{4}$ & 82 \\
${ }_{102}^{256} \mathrm{No} \rightarrow{ }_{51}^{128} \mathrm{Sb}+{ }_{51}^{128} \mathrm{Sb}$ & 110 & $0.9 \times 10^{-2}$ \\
${ }_{102}^{256} \mathrm{No} \rightarrow{ }_{46}^{116} \mathrm{Pd}+{ }_{56}^{140} \mathrm{Ba}$ & 110 & $0.3 \times 10^{-1}$ \\
\hline
\end{tabular}

The experimental spontaneous fission half-lives and theoretical predictions for the supposed most probable exit channels are compared in Table 2. The half-lives corresponding to several paths are given for ${ }^{243} \mathrm{Cm},{ }^{250} \mathrm{Cf},{ }^{256} \mathrm{Fm}$ and ${ }^{256} \mathrm{No}$. Except for the lightest $\mathrm{U}$ isotopes, there is a very correct agreement with the experimental 
data on 20 orders of magnitude. The half-lives vary regularly for close exit channels of a same nucleus.

\section{Summary and Conclusion}

Super- and hyperdeformed minima lodging possibly isomeric states appear for the actinide elements in the quasi-molecular shape path within a deformation energy derived from a generalized liquid drop model and including the shell and pairing energies. The second peak corresponds to the transition from one-body shapes to two touching ellipsoids. The third barrier appears only in the asymmetric decay path and for some specific nuclei. Then, the heaviest fragment is almost a magic nucleus and it preserves its shape close to the sphere. The other fragment evolves from an oblate ellipsoid to a prolate one and the third peak corresponds to the maximum of the shell effects in the non-magic fragment and, consequently, to two touching different spheres. The barrier heights agree precisely with the experimental results for the double and triple-humped fission barriers in all the actinide region. The predicted half-lives follow the experimental data trend on 20 orders of magnitude.

\section{References}

1. M.C. Duijvestijn, A.J. Koning and F.J. Hambsch, Phys. Rev. C64 (2001) 014607 .

2. A. Krasznahorkay, M. Hunyadi, M.N. Harakeh, M. Csatlós, T. Faestermann, A. Gollwitzer, G. Graw, J. Gulyás, D. Habs, R. Hertenberger, H.J. Maier, Z. Máté, D. Rudolph, P. Thirolf, J. Timár and B.D. Valnion, Phys. Rev. Lett. 80 (1998) 2073.

3. A. Krasznahorkay, D. Habs, M. Hunyadi, D. Gassmann, M. Csatlós, Y. Eisermann, T. Faestermann, G. Graw, J. Gulyás, R. Hertenberger, H.J. Maier, Z. Máté, A. Metz, J. Ott, P. Thirolf and S.Y. Van Der Werf, Phys. Lett. B461 (1999) 15.

4. M. Hunyadi, D. Gassmann, A. Krasznahorkay, D. Habs, P.G. Thirolf, M. Csatlós, Y. Eisermann, T. Faestermann, G. Graw, J. Gulyás, R. Hertenberger, H.J. Maier, Z. Máté, A. Metz and M.J. Chromik, Phys. Lett. B505 (2001) 27.

5. J. Blons, C. Mazur, D. Paya, M. Ribrag and H. Weigmann, Nucl. Phys. A414 (1984) 1.

6. A. Marinov, S. Gelberg, D. Kolb, R. Brandt and A. Pape, Int. J. Mod. Phys. E12 No. 5 (2003) 1.

7. A. Galindo-Uribarri, H.R. Andrews, G.C. Ball, T.E. Drake, V.P. Janzen, J.A. Kuehner, S.M. Mullins, L. Persson, D. Prévost, D.C. Radford, J.C. Waddington, D. Ward and R. Wyss, Phys. Rev. Lett. B71 (1993) 231.

8. G. Royer and F. Haddad, Phys. Rev. C47 (1993) 1302.

9. V.M. Strutinsky, Nucl. Phys. A95 (1967) 420. 
10. W.D. Myers and W.J. Swiatecki, Ark. Fys. 36 (1967) 343.

11. W.D. Myers, Droplet model of atomic nuclei, Plenum, New-York, 1977.

12. W.D. Myers and W.J. Swiatecki, Nucl. Phys. A601 (1996) 141.

13. M.G. Mustafa, U. Mosel and H.W. Schmitt, Phys. Rev. C7 (1973) 1519.

14. J.F. Berger, M. Girod and D. Gogny, J. Physique Lett. 42 (1981) 509.

15. V. Blum, J.A. Maruhn, P.G. Reinhard and W. Greiner, Phys. Lett. B323 (1994) 262.

16. S. Björnholm and J.E. Lynn, Rev. Mod. Phys. 52 (1980) 725.

17. C. Wagemans, The nuclear fission process, CRC Press, Boca Raton, 1991.

18. G. Royer and B. Remaud, J. Phys. G10 (1984) 1541.

19. G. Royer and F. Haddad, Phys. Rev. C51 (1995) 2813.

20. G. Royer and K. Zbiri, Nucl. Phys. A697 (2002) 630.

21. G. Royer, J. Phys. G26 (2000) 1149.

22. G. Royer and R. Moustabchir, Nucl. Phys. A683 (2001) 182.

23. C. Bonilla and G. Royer, Heavy Ion Phys. 17/1 (2003) 49.

24. G. Royer, C. Bonilla and R.A. Gherghescu, Phys. Rev. C67 (2003) 34315.

25. G. Royer and B. Remaud, Nucl. Phys. A444 (1985) 477.

26. G. Royer and C. Piller, J. Phys.: Nucl. Phys. G18 (1992) 1805. 